\title{
Mycoplasma californicum, a New Species from Cows
}

\author{
D. E. JASPER, ${ }^{1}$ H. ERN $\emptyset,{ }^{2}$ J. D. DELLINGER, ${ }^{1}$ AND C. CHRISTIANSEN ${ }^{2}$ \\ Department of Clinical Pathology, School of Veterinary Medicine, University of California, Davis, \\ California 95616, ${ }^{1}$ and Institute of Medical Microbiology, University of Aarhus, 8000 Aarhus C, Denmark ${ }^{2}$
}

Mycoplasmas serologically different from the known Mycoplasma species were found to be important causes of mastitis in California. We describe the cultural, morphological, biological, and serological characteristics of one of these isolates, strain ST-6. In growth inhibition and immunofluorescence tests, we found that strain ST-6 differed from all 75 currently accepted Mycoplasma species and serogroups. Therefore, we regard this strain as a member of a new species for which we propose the name Mycoplasma californicum. Strain ST-6, the type strain, has been deposited in the Collaborating Center for Animal Mycoplasmas, Institute of Medical Microbiology, University of Aarhus, Denmark, under the accession number AMRC-C 1077 and in the American Type Culture Collection under the number ATCC 33461.

In 1972, mycoplasmas of an unidentified species were isolated from a mastitis outbreak in California involving about 50 cows out of a herd of 350 (11). These organisms were distinguished from other Mycoplasma species by immunofluorescence (1). Strain ST-6 was designated the reference strain for this group of isolates. From December 1972 to June 1978 this organism was the sole mycoplasma identified from mastitis outbreaks in 17 herds and was one of two my. coplasmas found in another mastitis problem herd (13). From 1 July 1978 to 31 December 1979, eight more mastitis outbreaks were found to be due to ST-6-like mycoplasmas (12), and from 1 January 1980 to 30 June 1980, an additional 9 of 32 new outbreaks of mastitis were found to be due to the same organism. Thus far, 35 outbreaks have involved this mycoplasma.

The clinical disease caused by this organism is characterized by acute onset, variable swelling of the udder, slightly yellow, watery milk with a sandy sediment, flakes or clots or a seropurulent secretion, and either a marked reduction in milk flow or agalactiae. Occasional secretions may contain blood. Morbidity may be prolonged, but the organism tends to disappear with clinical recovery and may not always be recovered upon culture during the clinical stages.

In a preliminary study (5), Dellinger et al. found that strain ST-6 was serologically and biochemically different from 10 known Mycoplasma species of bovine origin. (Unfortunately, through a transcribing error during manuscript preparation, this organism was originally incorrectly reported as being arginine positive.) The present study was undertaken to determine whether strain ST-6 does indeed constitute a new Mycoplasma species.

\section{MATERIALS AND METHODS}

The recommendations of the Subcommittee on the Taxonomy of Mollicutes (17) were used as a basis for these studies and for our proposal that strain ST-6 be recognized as belonging to a new species.

Mycoplasma strains. Strain ST-6 was first isolated from a mastitis outbreak in 1972 (11) and was stored at $-70^{\circ} \mathrm{C}$. Before use, the cells were filter cloned five times through a membrane filter (pore size, 450 $\mathrm{nm}$ ). Two clone lines were selected and used for these taxonomic studies and for submission to culture collections. During the characterization studies, the cultures were stored at $4^{\circ} \mathrm{C}$ between serial passages.

The 75 reference cultures with which strain ST-6 was compared by immunofluorescence and growth inhibition tests were obtained from the FAO/WHO Collaborating Center for Animal Mycoplasmas, Institute of Medical Microbiology, University of Aarhus, Aarhus, Denmark. Table 1 shows the type and reference strains used for this comparison.

Medium. The standard medium which we used for primary isolations was essentially the modified Hayflick medium B used by Ernø and Stipkovits (7), but containing $2 \%$ (vol/vol) $1 \mathrm{M} \mathrm{K}_{2} \mathrm{HPO}_{4}$ buffer. For solid medium plates, $1.4 \%$ (wt/vol) agar (Difco Laboratories, Detroit, Mich.) was added. At times, transfer and maintenance of cultures were in tubes containing slants of $2 \mathrm{ml}$ of solid medium made with $5 \%$ horse blood overlaid with $3 \mathrm{ml}$ of liquid medium.

Morphology. Colonies were examined by light microscopy at $\times 25$ after incubation for 3 days at $37^{\circ} \mathrm{C}$ in $10 \% \mathrm{CO}_{2}$ in air in a humidified $\mathrm{CO}_{2}$ incubator.

Cells from 1-, 2-, and 3-day-old broth cultures were examined for morphology by oil immersion light microscopy $(\times 1,000)$ after cover glass preparations were stained with Wright-Leishman stain followed by Giemsa stain.

Thin sections were made of pellets from 2-day-old cultures and were examined with an electron microscope after fixation in $6.25 \%$ glutaraldehyde and then in $2.0 \%$ osmic acid.

Filterability. Filterability was determined with 1- 
TABLE 1. Strains of the 75 currently recognized Mycoplasma species and serogroups used for serological comparison

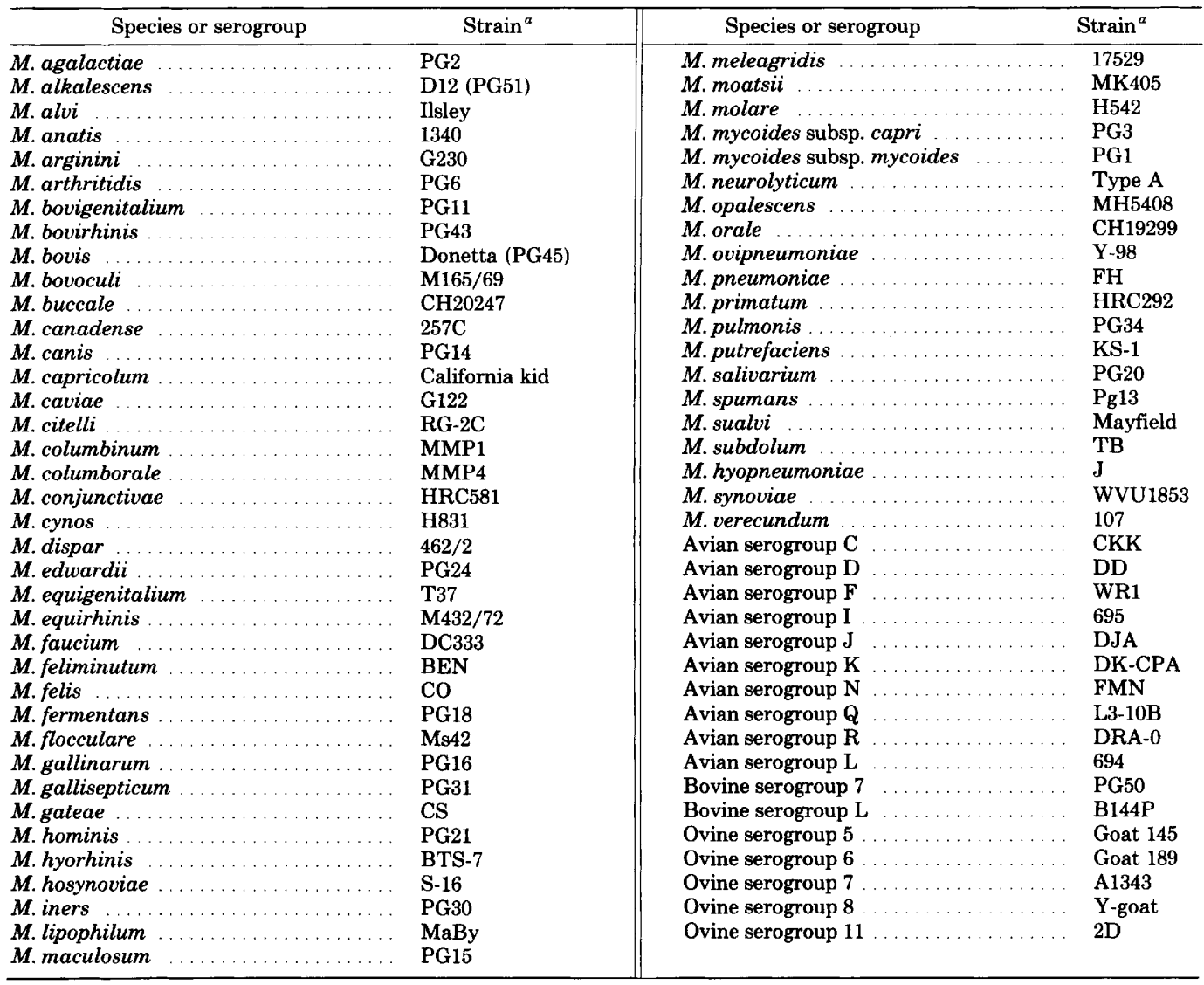

${ }^{a}$ All cultures were from the WHO/FAO Collaborative Center for Animal Mycoplasmas, University of Aarhus, Aarhus, Denmark.

and 2-day-old broth cultures. Filtrates were cultured by serial dilution into broth tubes and were incubated for 2 days at $37^{\circ} \mathrm{C}$ before they were subcultured onto agar plates.

Reversion. Reversion to bacterial forms was determined in the standard medium without inhibitors by five serial passages through broth media and one final passage on agar without bacterial inhibitors.

Cholesterol requirements. Cholesterol requirements were determined by the method of Razin and Tully (14), except that protein determinations were performed by the Bradford method (3) and records of colony counts were kept. Results were confirmed by the test for sensitivity to digitonin (7).

Biochemical characteristics. Tests for catabolism of glucose and arginine, hydrolysis of urea, and phosphatase activity were performed as previosuly described (8). Tests for glucose, arginine, and urea utilization were repeated by using FF II broth medium (7) as the base medium. Tests for aerobic catabolism of glucose were repeated by using $b_{1}$ and FF II media; observations for changes in glucose levels in the media were made at intervals by using the orthotoluidine method of Feteris (10). The triphenyl tetrazolium chloride reduction test was performed with $b_{1}$ and FF II media (8) under aerobic conditions. The following controls were used for these tests: uninoculated medium with substrate, inoculated medium without substrate, and organisms positive for each test.

Film and spot formation. Determinations of film and spot formation were made with medium BY of Fabricant and Freundt (9).

Serological tests. Hyperimmune sera against 74 type and reference strains were prepared in rabbits at the FAO/WHO Collaborating Center for Animal My. coplasmas (6). Goat antiserum against Mycoplasma sualvi Mayfield was kindly supplied by R. N. Gourlay, Animal Research Center Institute for Research on Animal Diseases, Compton, England. Strain ST-6 was tested with these sera (except $M$. sualvi antiserum, as anti-goat gamma globulin was not available) by the agar well modification of the growth inhibition technique of Black (2) and by the indirect immunofluorescence technique, as described by Rosendal and Black (15). Hyperimmune serum against strain ST-6 was tested against the homologous strain and against all 
75 type and reference strains (except $M$. sualvi Mayfield, as we were unable to grow this strain) by the methods described above.

Deoxyribonucleic acid base composition. Deoxyribonucleic acid was extracted as described previously (4). The guanine-plus-cytosine content of the deoxyribonucleic acid was determined by analytical centrifugation in $\mathrm{CsCl}$, using deoxyribonucleic acid $\left.1.7100 \mathrm{~g} / \mathrm{cm}^{3}\right)(4,16)$ from Escherichia coli as a reference.

\section{RESULTS}

Morphology. Compared with some other species of bovine origin, the growth of strain ST6 was somewhat slower on the media which we used. After $72 \mathrm{~h}$ of growth, the maximum colony size was usually about $0.3 \mathrm{~mm}$ for well-separated colonies having the classical fried egg appearance. Smaller colonies were conical and had distinct small centers (Fig. 1). Larger colonies appeared to be conical but were more rounded and also had distinct small centers (Fig. 1). Colony surfaces appeared to be smooth but had a slight granularity around the center.

Examinations of stained films of growth in broth after 1,2 , and 3 days showed many pleomorphic organisms (small coccoid, filamentous, ring, and conglomerate forms) (Fig. 2). Small coccoid forms were barely visible. Ring forms were more common in some preparations, and small filamentous forms were more common in others, but this did not appear to be related to the age of the culture. Electron microscopy revealed pleomorphic cells surrounded by the three-layered membranes which are typical of mycoplasmas (Fig. 3).

Filterability. Neither 1- nor 2-day-old broth cultures of strain ST-6 were filterable at $220 \mathrm{~nm}$. Both cultures were readily filterable at $450 \mathrm{~nm}$.
The number of colony-forming units in the 24h-old cultures was less than the initial count $\left(10^{8}\right.$ colony-forming units per $\mathrm{ml}$ ) by 2 to 4 logs. The corresponding decrease in colony-forming units for the 48-h-old cultures was somewhat greater (4 to 5 logs).

Reversion. Reversion to bacterial colony types did not occur.

Cholesterol requirements. The organisms tested failed to grow in serum-free medium, as shown by protein yields and colony growth (Table 2). The growth of strain ST-6 was similar in these respects to that of Mycoplasma bovis but was not as abundant. Rapid die-off in a medium containing $15 \%$ horse serum was observed. Growth did not occur in the presence of digitonin.

Biochemical characteristics. Strain ST-6 was phosphatase positive but failed to reduce

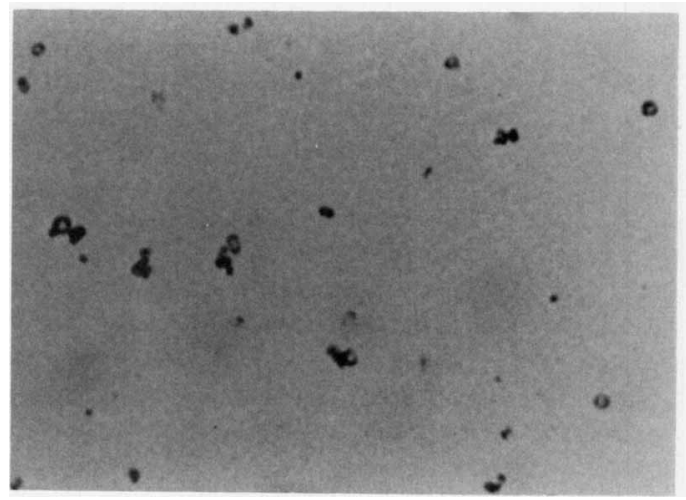

FIG. 2. M. californicum strain ST'6 broth culture cells stained with Wright-Leishman and Giemsa stains.

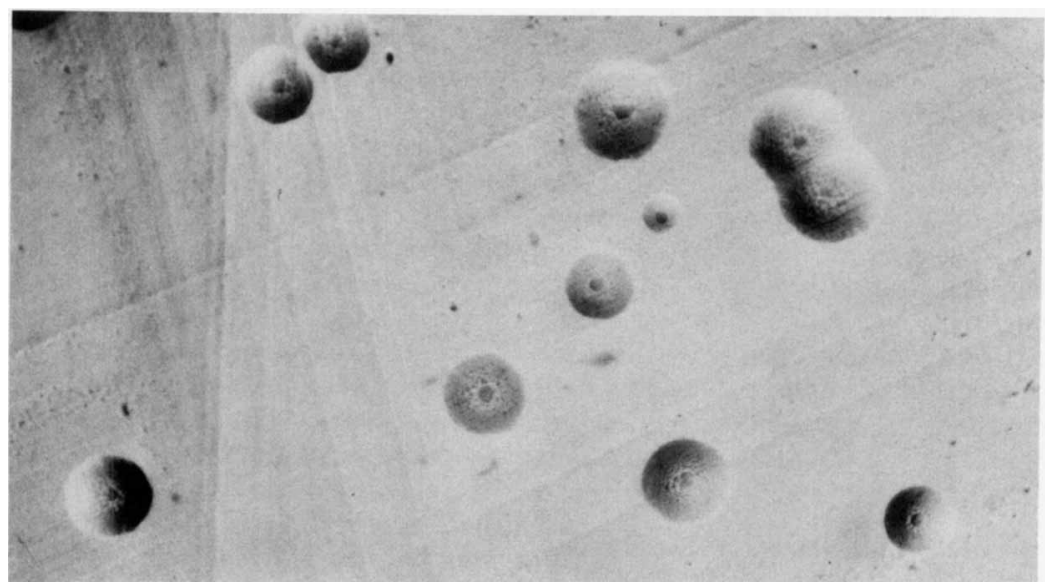

Fig. 1. M. californicum strain $S T-6: 72-h$-old colonies on agar. $\times 25$. 




FIG. 3. Electron micrograph of a broth culture pellet of M. californicum strain $S T-6 . \times 47,500$.

triphenyl tetrazolium chloride aerobically and did not ferment glucose aerobically in any of the tests used. Neither arginine nor urea was hydrolyzed.

Film and spots. Film and spots were not produced.

Serological tests. Strain ST-6 was tested with hyperimmune sera against 75 currently accepted Mycoplasma species and serogroups by the growth inhibition and indirect immunofluorescence techniques. Cross-reactions were not observed in the indirect immunofluorescence tests, although antiserum against $M$. sualvi slightly inhibited strain ST-6.

Antiserum against ST-6 inhibited Mycoplasma fermentans, Mycoplasma maculosum, Mycoplasma meleagridis, and Mycoplasma synoviae (Table 3 ), but all immunofluorescence tests were negative.

Guanine-plus-cytosine content of deoxyribonucleic acid. The buoyant density of the deoxyribonucleic acid was $1.691 \mathrm{~g} / \mathrm{cm}^{3}$, which was equivalent to a guanine-plus-cytosine content of $31.9 \mathrm{~mol} \%$.

\section{DISCUSSION}

The findings described above indicate that the organism designated strain ST-6 meets the re- quirements for classification with the class $\mathrm{Mol}$ licutes, order Mycoplamatales, family Mycoplasmataceae, and genus Mycoplasma (17). The physical, morphological, and cultural characteristics of strain ST- 6 are consistent with those of the Mycoplasmatales (17). The serum and cholesterol requirements of this strain support its classification as a member of the family $\mathrm{Myco}$ plasmataceae. The growth inhibition and immunofluorescence tests demonstrated that strain ST-6 is serologically different from the 75 currently recognized Mycoplasma species and serogroups listed in Table 1 . The rapid die-off observed in media containing $15 \%$ horse serum is consistent with long-term laboratory observations that strain ST-6 cultures may be lost if they are not transferred in time, but this does not always occur within 4 days.

Serological tests comparing strain ST-6 with type and reference strains of the currently recognized Mycoplasma species and some serogroups with their hyperimmune sera have revealed that strain ST-6 represents a new species. No cross-reactions were observed in the immunofluorescence tests, and since the few crossreactions which were observed in the growth inhibiton tests were one way, they were considered to be nonspecific. The growth inhibition of 


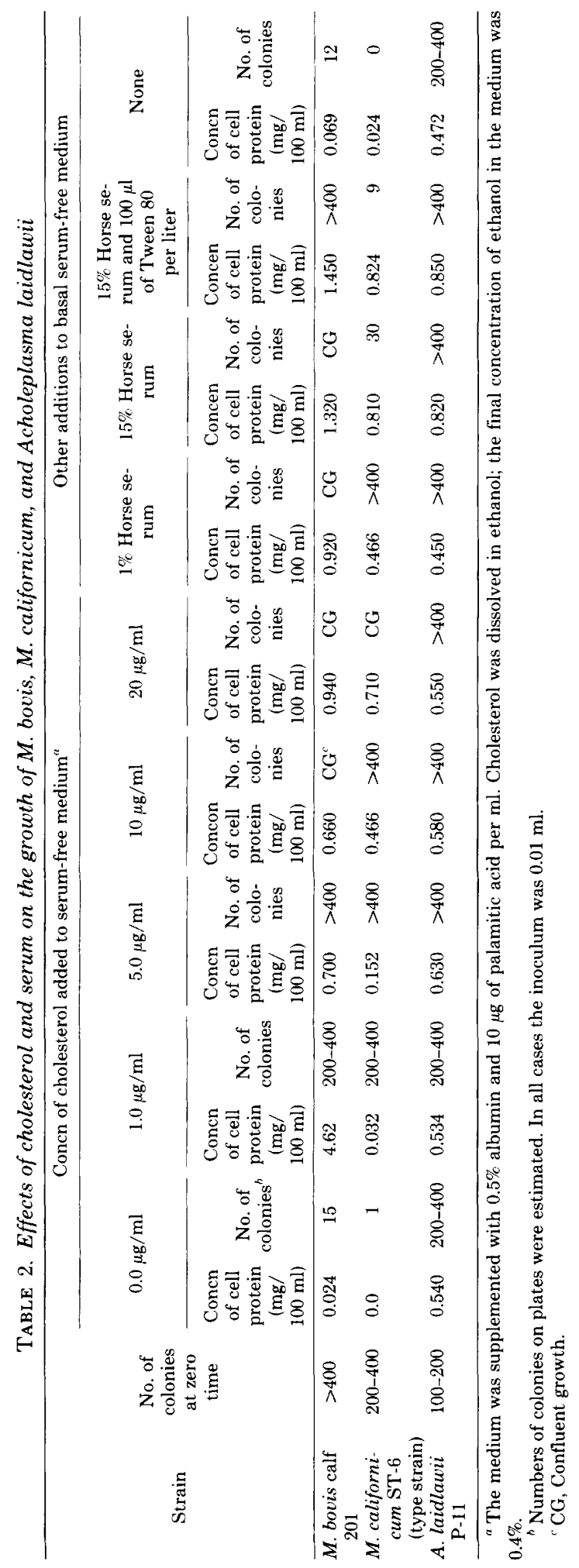


TABLE 3. Growth inhibition tests showing the cross-reactions between strain ST-6 and other Mycoplasma strains

\begin{tabular}{llllllll}
\hline \multirow{2}{*}{ Species } & \multirow{2}{*}{ Strain } & \multicolumn{5}{c}{ Diam $(\mathrm{mm})$ of inhibition zone with the following antisera: } \\
\cline { 3 - 8 } & & ST-6 & Mayfield & PG18 & PG15 & 17529 & WVU1853 \\
\hline Not designated & ST-6 & 9 & $3.5(\mathrm{NT})^{a}$ & 0 & 0 & 0 & 0 \\
$M$. sualvi & Mayfield & $\mathrm{ND}^{b}$ & $\mathrm{ND}$ & $\mathrm{ND}$ & $\mathrm{ND}$ & ND & ND \\
$M$. fermentans & PG18 & 1.5 & 0 & 8 & 0 & 0 & 0 \\
$M$. maculosum & PG15 & 5 & 0 & 0 & 7 & 0 & 0 \\
$M$. meleagridis & 17529 & 6 & 0 & 0 & 0 & 8 & 0 \\
$M$. synoviae & WVU1853 & 1 & 0 & 0 & 0 & 0 & 7 \\
\hline
\end{tabular}

${ }^{a}$ NT, Not total (the zone of inhibition was not total, but the number of "break-through" colonies was less than 10 ).

${ }^{b} \mathrm{ND}$, Not done.

strain ST-6 by $M$. sualvi antiserum is also considered nonspecific, as strain ST-6 and the type strain of $M$. sualvi (strain Mayfield) are different biochemically as well as in their growth requirements.

Because thus far this mycoplasma has been encountered only in California and has been described as a frequent cause of bovine mastitis in that state, we propose the name Mycoplasma californicum (cal.i.for'ni.cum. M.L. adj. californicus pertaining to California) for this species.

The following is a description of $M$. californicum sp. nov.

Morphology and staining characteristics: minute coccoid, filamentous, ring, conglomerate, and other pleomorphic forms. Staining is dark blue with Wright-Leishman stain plus Giemsa stain. The cells are bounded by a single triplelayered cell membrane.

Colonies reach about $0.3 \mathrm{~mm}$ in diameter, are somewhat conical in shape, and have a typical fried egg appearance.

Viable cells are readily filtered at $450 \mathrm{~nm}$ but are not filterable at $220 \mathrm{~nm}$.

Liquid cultures are slightly turbid.

Film and spots are not formed on modified Hayflick medium containing horse serum or $10 \%$ egg yolk agar.

Biochemical reactions: glucose is not utilized, and arginine and urea are not hydrolyzed. Triphenyl tetrazolium chloride is not reduced aerobically, but the phosphatase test is positive.

No growth occurs in standard mycoplasma medium without serum. Cholesterol is required.

Pathogenicity: causes bovine mastitis.

Habitat: bovine udders with mastitis. The normal habitat is unknown.

The type strain of $M$. californicum is strain ST-6, which was isolated in 1972 from the first outbreak of mastitis found to be due to this organism. Cultures of this strain have been deposited in the Collaborating Center for Animal Mycoplasmas, Institute of Medical Microbiol- ogy, University of Aarhus, Aarhus, Denmark, under the number AMRC-C 1077 and in the American Type Culture Collection under the number ATCC 33461.

\section{ACKNOWLEDGMENTS}

This work was supported in part by the California Milk Advisory Board and the U.S. Department of Agriculture [ARS cooperative agreement 12-14-100-2380(45)].

\section{REPRINT REQUESTS}

Address reprint requests to: Dr. D. E. Jasper, Department of Clinical Pathology, School of Veterinary Medicine, University of California, Davis, CA 95616.

\section{LITERATURE CITED}

1. Baas, E. J., and D. E. Jasper. 1972. Agar block technique for identification of mycoplasmas by use of fluorescent antibody. Appl. Microbiol. 23:1097-1100.

2. Black, F. T. 1973. Modifications of the growth inhibition test and its application to human T-mycoplasmas. Appl. Microbiol. 25:528-533.

3. Bradford, M. M. 1976. A rapid and sensitive method for the quantitation of microgram quantities of protein utilizing the principle of protein-dye binding. Anal. Biochem. 72:248-254.

4. Christiansen, C., E. A. Freundt, and F. T. Black. 1975 Genome size and deoxyribonucleic acid base composition of Thermoplasma acidophilum. Int. J. Syst. Bacteriol. 25:99-101.

5. Dellinger, J. D., D. E. Jasper, and M. Ilić. 1977. Characterization studies on mycoplasmas isolated from bovine mastitis and the bovine respiratory tract. Cornell Vet. 67:351-360.

6. Ernø, H., K. Jurmanová, and R. H. Leach. 1973. Bovine mycoplasmas: a serological study by the metabolic inhibition test. Acta Vet. Scand. 14:511-523.

7. Ernø, H., and L. Stipkovits. 1973. Bovine mycoplasmas: cultural and biochemical studies. I. Acta Vet. Scand. 14:436-449.

8. Ernø, H., and L. Stipkovits. 1973. Bovine mycoplasmas: cultural and biochemical studies. II. Acta Vet. Scand. 14:450-463.

9. Fabricant, J., and E. A. Freundt. 1967. Importance of extension and standardization of laboratory tests for the identification and classification of mycoplasmas. Ann. N. Y. Acad. Sci. 143:50-58.

10. Feteris, A. 1965. A serum glucose method without protein precipitation. Am. J. Med. Technol. 31:17-20.

11. Jasper, D. E. 1977. Mycoplasma and mycoplasma mas- 
titis. J. Am. Vet. Med. Assoc. 170:1167-1172.

12. Jasper, D. E. 1980. Prevalence of mycoplasmal mastitis in the western states. Calif. Vet. 33:24-26.

13. Jasper, D. E., J. D. Dellinger, M. H. Rollins, and H. D. Hakanson. 1979. Prevalence of mycoplasmal bovine mastitis in California. Am. J. Vet. Res. 40:1043-1047.

14. Raxin, S., and J. G. Tully. 1970. Cholesterol requirement of mycoplasmas. J. Bacteriol. 102:306-310.

15. Rosendal, S., and F. T. Black. 1972. Direct and indirect immunofluorescence of unfixed and fixed mycoplasma colonies. Acta Pathol. Microbiol. Scand. Sect. B. 80: 615-622.

16. Schildkraut, C. L., J. Marmur, and P. Doty. 1962. Determination of the base composition of deoxyribonucleic acid from its buoyant density in $\mathrm{CsCl}$. J. Mol. Biol. 4:430-443.

17. Subcommittee on the Taxonomy of Mollicutes. 1979. Proposal of minimal standards for descriptions of new species of the class Mollicutes. Int. J. Syst. Bacteriol. 29:172-180. 